\title{
Treatment Outcome of Severe Acute Malnutrition Among Children (6-59 Months) in Outpatient Therapeutic Feeding Program in Gursum District, Somali Region, Ethiopia
}

\author{
Abdulahi Bedel Budul ${ }^{*}$, Alinoor Mohamed Farah, Tahir Yusuf Nour \\ Department of Public Health, Jigjiga University, Jigjiga, Ethiopia \\ Email address: \\ abdulahi.bbh@gmail.com (A. B. Budul), alinuriana@yahoo.com (A. M. Farah), daahir571@gmail.com (T. Y. Nour) \\ ${ }^{*}$ Corresponding author \\ To cite this article: \\ Abdulahi Bedel Budul, Alinoor Mohamed Farah, Tahir Yusuf Nour. Treatment Outcome of Severe Acute Malnutrition Among Children (6- \\ 59 Months) in Outpatient Therapeutic Feeding Program in Gursum District, Somali Region, Ethiopia. Science Journal of Public Health. \\ Vol. 8, No. 2, 2020, pp. 36-42. doi: 10.11648/j.sjph.20200802.12
}

Received: February 5, 2020; Accepted: February 27, 2020; Published: March 17, 2020

\begin{abstract}
Severe Acute Malnutrition (SAM) is one of public health problems in developing countries which mainly affect under five children. Ethiopia is one of the countries with uppermost under-five child mortality rate, in which malnutrition contributes to $57 \%$ of all children's death. This study provides an insight based on management of SAM and help to improve the management of severe acute malnutrition in outpatient therapeutic program (OTP). This study aimed to assess treatment outcomes of severe acute malnutrition and associated factors among children treated at outpatient program in Gursum woreda, Eastern Ethiopia. Facility-based retrospective cross-sectional study was conducted in outpatient program records of 350 children, treated at twelve health facilities obtained from January 1, 2016 to December 31, 2018. Proportional allocation was conducted to get sample size for each selected health facility and outpatient program sites within woreda. Individual cards of children were selected by systematic random sampling. Data were entered using Epi data software version 3.1 and exported to SPSS version 22 for cleaning and further analysis. Crude and Adjusted odd ratio along with the $95 \%$ confidence interval were estimated by using bivariate and multivariate logistic regressions to identify factors associated with treatment outcome. Level of significance was declared at P-value less or equal to 0.25 and 0.05 respectively. The recovery rate of sever acute malnutrition was $81.7 \%$. Death rate, defaulter rate, weight gain, and length of stay were $0 \%, 12.6 \%, 3.8 \mathrm{~g} / \mathrm{kg} / \mathrm{day}$ and 6.7 weeks respectively. The odd of recovery was 2.5 times higher for children with kwashiorkor than children with marasmus (AOR: $2.877,95 \%$ CI: $1.141,7.255, \mathrm{P}=0.025$ ), and those children with self-referral were $57.4 \%$ more likely to recover than their counterpart. The average weight gain and length of stay were not in acceptable range of international SPHERE Standard. Also Type of malnutrition and self-referral were factors identified as significant associated with treatment outcome of severe acute malnutrition. Capacity building of OTP service providers, regular monitoring of service provision based on the severe acute management protocol and awareness creation of community were recommended.
\end{abstract}

Keywords: Severe Acute Malnutrition, Treatment Outcome, Outpatient Therapeutic Feeding, Program, Somali Region, Eastern Ethiopia

\section{Introduction}

Acute malnutrition is due to inadequate nutritional intake that often coupled with infections [1]. World Health Organization (WHO) 2013 guideline, defined Severe Acute Malnutrition is when $\mathrm{WHZ}<-3$, MUAC $<11.5 \mathrm{~cm}$, and $/$ or presence of bilateral edema [2]. Based on their severity acute malnutrition it is classified as Moderate Acute Malnutrition
(MAM) and Severe Acute Malnutrition (SAM). Furthermore, SAM is subdivided into SAM with complication \& SAM without complication $[1,3]$.

SAM threatens the survival of children less than five years of age both in emergency and non-emergency settings, globally it affects 20 million under five children while one million of them die yearly. In Sub-Saharan Africa severe acute malnutrition contribute 1.7 million under five children, 
SAM children are nine times more at risk of dying than healthy children $[1,4]$.

According to Ethiopian Demographic and Health Survey (EDHS) 2016, nationally children that are wasted, underweight, and stunted were $10 \%, 24 \%$ and $38 \%$ respectively. When the data is segregated into regions, Somali region has the highest wasting prevalence; $22.7 \%$ and with underweight and stunting being $28.7 \%$ and $27.4 \%$ respectively [5].

Previously SAM treatment was provided only at health facility based on therapeutic feeding center (TFC) which had its own drawback like length of stay for inpatient treatment. The innovative community-based approach was success and scaled up SAM treatment program and made it possible to treat majority of children at their home, and in 2017 it was endorsed by United Nations [6-8].

This decentralized community management of acute malnutrition (CMAM) program components covers community outreach, OTP, SC and TSFP. OTP serves management of children aged 6-59 month with admission criteria of MUAC <11.5, nutritional edema is $+/++$, passed the appetite test, and no medical complications. Ready-to-use therapeutic foods (RUTF), and routine medications like antibiotics, vitamin A, folic acid, deworming, anti-malaria, and measles vaccine are used to manage acute malnutrition with no medical complications [6, 8-11]. Based on SAM management protocol children obtain weekly RUFT and routine supportive therapies based on their weight and discharged by their admission criteria and required target whether it is MUAC WFH\% or absence of Edema for two consecutive weeks $[9,12]$.

According to united nation international children's emergency fund (UNICEF) in 2015, approximately 2.9 million children accessed treatment in 65 countries in 2013 that is only about 17 per cent of the children needing treatment [13]. Ethiopia is one of the countries with highest under five child mortality, which malnutrition accounts about $57 \%$ of all children deaths, despite that SAM is critical for child survival and it is cost-effective to scale up community based nutrition intervention, Ethiopian government is struggling to provide sufficient resource to prevent and treat acute malnutrition [14]. Most of the children with SAM are sent to hospitals or health centre to be treated at TFU and OTP. Though major determinant factors for poor treatment outcome are not well understood, factors like delay in seeking care, co-morbidities, poor case management are some of the factors identified [15].

Therefore, the current work will assess treatment outcome of severe acute malnutrition and associated factors among children (6-59 months) treated at outpatient therapeutic feeding program in Gursum woreda, Somali Region, Ethiopia. The current work will help to provide clue on pastoralist regions to develop program intervention which suit them for long term planning particularly for CMAM Programme. Though there are studies which have been conducted in other part of the country they focused mainly on inpatient programs and mainly conducted in the highlands of
Ethiopia where the context is purely agrarian and unlike previous studies, the current was conducted in pastoralist context.

\section{Method}

\subsection{Study Area, Period, Design}

Institutional based retrospective cross-sectional study was conducted Gursum district of Fafan zone, Somali regional state from January 1, 2016 to December 31, 2018. It has geographical relationship with Babili on Southwest Oromo Region, North Ajersagora, on East Jigjiga, and on the Southeast Mulla district, based on the 2007 Census conducted by the Central Statistical Agency of Ethiopia (CSA), this district has a total population of 27,510, of whom 14,815 are men and 12,695 women and under five children is 3,879 , While 2,970 or $10.8 \%$ are urban inhabitants, a further 2,028 or $7.37 \%$ are pastoralists [16]. The woreda consist 22 main kebeles, having of 22 health facilities ( 3 health centres \& 19 health posts) which are providing therapeutic feeding program and care Ethiopia is only partner supporting in Gursum woreda.

\subsection{Source and study Population}

All records of children (6-59 months) treated an outpatient therapeutic feeding program in Gursum woreda. Records of children selected by systematic random sampling from randomly selected outpatient therapeutic feeding program sites, from January 1, 2016 up to December31, 2018, was the study population.

\subsection{Inclusion and Exclusion Criteria}

Records of children admitted to OTP from January1, 2016 up to December31, 2018 were included; children those readmitted, incomplete records were excluded.

\subsection{Sample Size Determination}

Sample size was calculated using a single population proportion formula with the following assumptions of recovery rate (cured) among under five children with severe acute malnutrition to be $64.9 \%$ [17], then the sample size shows as below.

$$
\mathrm{N}=\mathrm{Z}^{2} \mathrm{P}(1-\mathrm{P}) / \mathrm{d}^{2}
$$

Where, $\mathrm{n}=$ sample size derived from estimation formula $Z^{2-\alpha / 2}=$ is confidence interval, i.e. 1.96 to be $95 \%$ confident

$\mathrm{P}=$ is recovery rates of children who had been managed for SAM under OTP

$\mathrm{d}=\mathrm{is}$ margin of error to be tolerated and taken as $5 \%$

$$
\mathrm{N}=\left(1.96^{2}\right)\left(0.649(1-0.649) /\left(0.05^{2}\right)\right.
$$

\subsection{Sampling Procedure}

In Gursum woreda there are twenty-two health facilities 
which are providing (OTP) services. Twelve health facilities were selected randomly using lottery method presuming that there was no information lost with unselected health facilities applying OTP protocol for management of severe acute malnutrition that works equally to health facility level. Study population was selected using systematic random sampling. All records of children who attended in selected health facilities from January 1, 2016 to December 31, 2018 were listed based on sequence of unique identification number. Then study units were selected through proportional allocation to the size of each health facility by calculating the $\mathrm{N}=1423$ and sample size $n(K=N / n)$. The interval $(K=4)$ was similar each health facility and the first number to start was selected randomly. Total sample size was 350 it was divided proportionally in to the health facilities those providing OTP service in Gursum Woreda. Incomplete records were substituted to the nearest card number during data collection period.

\subsection{Data Collection}

Structured and pretested data collection template [17] was used to collect data from children OTP card and registration books, the records was reviewed mainly from children cards, and any missing variables in card was checked from registration book. Data collectors were diploma nurses from selected woreda, three full day training was given both data collectors; training was given on how to collect the data from the cards and filling the questionnaires in detail and demonstrated before they collected data. Two supervisors with the qualification of BSc in nursing from the woreda were involved after having two days training.

\subsection{Study Variables}

\subsubsection{Dependent Variable}

Treatment outcome.

\subsubsection{Independent Variables}

Socio-demographic characteristic: Age, sex,

Co- morbidities: Diarrhoea, cough, fever, anaemia,

Types of malnutrition: Wasting, oedematous,

Admission type: New, relapse,

Routine medication: Amoxicillin, deworming, vitamin A, measles vaccine, folic acid, anti-malaria,

Distance from health facility: less than 30 minutes, greater than or equal to 30 minutes,

Anthropometric measurements: Weight/height/length,

MUAC, Oedema,

Weight gain: $\geq 8 \mathrm{~g} / \mathrm{kg} /$ day, $<8 \mathrm{~g} / \mathrm{kg} /$ day,

Length of stay: $<4$ weeks, $>6$ weeks.

\subsection{Operational Definitions}

Adequacy of RUTF provision: Sufficiency of RUTF was calculated based on the national SAM management protocol standing from weight of respective children [18].

Co-morbidity: Additional medical problem with severe acute malnutrition [18].
Distance to OTP site: - Time taken from OTP site to house/residence of children by foot in minutes $/ \mathrm{km}$ [18].

New admission: Patient that are directly admitted to therapeutic feeding centre to start a nutritional treatment [18].

Not recovered: Defined as children discharged from OTP with outcome other than recovery in this study (death, default, non-responder, medical transfer, and falsely recovered). [18].

Recovered: Children attained 15\% weight gain from admission weight for children admitted with wasting and after two consecutive weeks from oedema disappeared for oedematous children [18].

Treatment outcome: Grouped as recovered and not recovered from SAM management at OTP in this study [18].

Type of malnutrition: - Grouped as marasmus (Wasting) and kwashiorkor (oedematous) [18].

Length of stay: - Number of days that the child stays in the outpatient therapeutic feeding program.

Weight gain: - An increase in weight of the child after being admitted in the outpatient therapeutic feeding program.

\subsection{Data Quality Control}

To ensure data quality, pre-test was conducted on $5 \%$ of the total sample outside the selected sites and close supervision was made at the time of data collection and to ensure the completeness, accuracy and consistency of information during data collection. The principal investigator and supervisors made thorough check before receiving the filled questionnaires from each data collector and in the meantime random cards were selected to cross check whether they were properly filled or not. Also, on spot visit was done during the data collection and the data collectors were debriefed on the observed findings. After data collection, the data was carefully entered in to Epi data version 3.1, cleaned, coded and analysed in to SPSS version 22.

\subsection{Data Processing and Analysis}

Data was analysed using SPSS version 22. The treatment outcomes were described by recovery, death, default, nonresponders for treatment, medical transfer and unknown rates. Both weight gain and length of stay was also calculated based on national protocol. Later, those predictor variables which had p-value of less than 0.25 in bivariate logistic regression were run in multivariate to identify the effect of each explanatory variable on the outcome variables. A pvalue less than 0.05 was considered as statistically significant variables and adjusted Odds ratio with $95 \%$ CI was calculated to identify any association.

\section{Results}

\subsection{Demographic Characteristics of Children}

The study included 350 eligible children records that had been managed for SAM in the OTP from January1, 2016 to December 31, 2018. A response rate of $100 \%$ was achieved 
and of patient records reviewed, $185(53 \%)$ of the children were males, 319 (91.1\%) were less than 24 months. The mean age at admission was 12 months ( $\mathrm{SD}= \pm 7.5$ months). Self-referred children at the program accounted $253(72.3 \%)$, whereas $209(59.7 \%)$ of children took less than 30 minutes to reach the health facilities (Table 1).

Table 1. Demographic characteristics of children admitted to OTP in Gursum woreda health facilities from 2016-2018.

\begin{tabular}{llll}
\hline Variables & Categories & Frequencies & Percentage (\%) \\
\hline \multirow{2}{*}{ Age } & $<24$ month & 319 & 91.1 \\
& $\geq 24$ month & 31 & 8.9 \\
Sex & Male & 185 & 53 \\
& Female & 165 & 47 \\
\multirow{2}{*}{ Referral } & Community voluntaries & 75 & 21.4 \\
system & self-referred & 253 & 72.3 \\
& Neighbors & 13 & 3.7 \\
Distance & EOS & 9 & 2.6 \\
from OTP & $>30$ minute & 209 & 59.7 \\
\hline
\end{tabular}

\subsection{Types of Malnutrition and Co-morbidities at Admission}

Regarding children admitted in to the OTP, only 37 $(10.6 \%)$ of them were admitted based on edema, where 313 (89.4\%) admitted on MUAC. Marasmic children accounted $313(89.4 \%)$, the rest were suffering from kwashiorkor 37 $(10.6 \%)$. For co-morbidity, among three hundred fifty children admitted to the program, 157 (44.9\%) children had cough, $98(27.7 \%)$ of them had diarrhea while $45(12.9 \%)$ had fever and 16 (4.6\%) were anemic (Table 2).

Table 2. Types of malnutrition and co-morbidities at admission in children admitted at OTP in Gursum woreda health facilities, from 2016-2018.

\begin{tabular}{llll}
\hline Variables & Categories & Frequencies & Percentage (\%) \\
\hline Type of & Marasmus & 313 & 89.4 \\
malnutrition & Kwashiorkor & 37 & 10.6 \\
Presence of & Yes & 37 & 10.6 \\
Edema & No & 313 & 89.4 \\
Presence of & Yes & 157 & 44.9 \\
cough & No & 193 & 55.1 \\
Presence of fever & Yes & 45 & 12.9 \\
& No & 305 & 87.1 \\
Presence of & Yes & 97 & 27.7 \\
diarrhea & No & 253 & 72.3 \\
Presence of & Yes & 16 & 4.6 \\
Anemia & No & 334 & 95.4 \\
Appetite test & Yes & 347 & 99.1 \\
done & No & 3 & 0.9 \\
Breast feed & Yes & 132 & 37.7 \\
\hline
\end{tabular}

\subsection{RUTF and Routine Medication Provision}

RUTF provided based on weight of children and majority of the children $338(96.6 \%)$ were provided with adequate amount of RUTF and as per the protocol. Routine medications were also provided at OTP for some children in the same program. Of all children provided with routine medication, 327 (93.4\%) were given amoxicillin, 200 (57.1\%) were supplemented with vitamin A, 160 (45.7\%) were vaccinated on measles vaccine and $52(14.9 \%)$ were provided with deworming tablets (Table 3).

Table 3. Provision of RUTF and Routine medications of children admitted to OTP in Gursum woreda health facilities from 2016-2018.

\begin{tabular}{llll}
\hline Variables & Categories & Frequencies & Percentage (\%) \\
\hline \multirow{2}{*}{ RUFT given } & Adequate & 338 & 96.6 \\
Amoxicillin & Inadequate & 12 & 3.4 \\
provision & Yes & 327 & 93.4 \\
Vit. A intake & No & 23 & 6.6 \\
& Yes & 200 & 57.1 \\
Deworming & No & 150 & 42.9 \\
given & Yes & 52 & 14.9 \\
Folic acid given & No & 298 & 85.1 \\
Vaccinated on & No & 5 & 1.4 \\
measles vaccine & No & 345 & 98.6 \\
Antimalaria & Yes & 160 & 45.7 \\
given & No & 190 & 54.3 \\
\hline
\end{tabular}

\subsection{Treatment Outcomes}

Out of 350 enrolled children with SAM in the OTP services, the recovery rate for SAM in OTP was $286(81.7 \%)$ and children recovered within $\leq 8$ weeks was277 (79.1\%). There was no reported death, but the defaulter rate, nonresponder rate, medical transfer, and unknown were 44 $(12.6 \%), 3(0.9 \%), 8(2.3 \%)$, and $9(2.6 \%)$, respectively, in this study. The average length of stay at OTP was 6.7 weeks, and children gain weight in average of $3.8 \mathrm{~g} / \mathrm{kg} /$ day for length of stay (Table4).

Table 4. Performance indicators of OTP and sphere standard references of Gursum woreda health facilities from 2016-2018.

\begin{tabular}{lll|l}
\hline \multirow{2}{*}{$\begin{array}{l}\text { Performance } \\
\text { indicators }\end{array}$} & $\begin{array}{l}\text { Frequencies of } \\
\text { indicators }\end{array}$ & \multicolumn{2}{l}{$\begin{array}{l}\text { International sphere } \\
\text { standards reference }\end{array}$} \\
\cline { 3 - 4 } & $286(81.7)$ & $>75 \%$ & $<50 \%$ \\
& $44(12.6)$ & $<15 \%$ & $>25 \%$ \\
\hline Recovery rate & $0(0 \%)$ & $<10 \%$ & $>15 \%$ \\
Defaulter & $3.8 \mathrm{~g} / \mathrm{kg} /$ day & $\geq 8 \mathrm{~g} / \mathrm{kg} / \mathrm{day}$ & $<8 \mathrm{~g} / \mathrm{kg} / \mathrm{da}$ \\
Death rate & & $<4$ weeks & $>6$ weeks \\
Average weight gain & 6.7 weeks & & \\
Average Length of stay & & & alarming \\
\hline
\end{tabular}

\subsection{Bivariate and Multivariate Logistic Regression Analysis of Treatment Outcome of Severe Acute Malnutrition Children Admitted to OTP in Gursum Woreda from $2016-2018$}

Bivariate and multivariate analysis was done to identify applicant variables for logistic regression. Those of $\mathrm{p}$ value $<0.25$ during bivariate logistic regression were selected as candidate for multivariate logistic regression; those with $\mathrm{p}$ value $<0.05$ were considered as significantly associated with outcome variable those variables were types of malnutrition and referral system.

Children suffering from kwashiorkor were 2.5 times more likely to recover from sever acute malnutrition compared to those with marasmus (AOR: 2.53, 95\% CI: 1.07, 6.03). Children that were self-referral were $58 \%$ less likely not to recover from severe acute malnutrition compared to their counterpart (AOR: 0.42, 95\% CI: 0.21, 0.865). We have 
found no relationship between child's age, sex, distance from OTP, RUTF intake, appetite test, breastfeeding and history of diarahea with the treatment outcome (Table 5).

Table 5. Results of Multivariable analysis of factors associated treatment outcome of children in OTP, Gursum woreda health facilities from 2016 - 2018.

\begin{tabular}{|c|c|c|c|c|c|c|c|}
\hline \multirow[b]{2}{*}{ Variables } & \multirow[b]{2}{*}{ Category } & \multicolumn{2}{|c|}{ Treatment outcome } & \multirow[b]{2}{*}{ COR 95\% (CI) } & \multirow[b]{2}{*}{ P-value } & \multirow[b]{2}{*}{ AOR (95\% (CI) } & \multirow[b]{2}{*}{ P-value } \\
\hline & & $\begin{array}{l}\text { Recovered } \\
(\%)\end{array}$ & $\begin{array}{l}\text { Not recovered } \\
(\%)\end{array}$ & & & & \\
\hline \multirow[b]{2}{*}{ Age } & $<24$ month & $234(81.3)$ & $54(18.8)$ & $1.200(0.573,2.512)$ & 0.629 & & \\
\hline & $>=24$ month & $52(83.9)$ & $10(16.1)$ & $1: 00$ & & & \\
\hline \multirow{2}{*}{ Sex } & Male & $157(84.9)$ & $28(15.1)$ & $0.639(0.370,1.103)$ & 0.108 & $0.615(0.331,1.141)$ & 0.123 \\
\hline & Female & $129(78.2)$ & $36(21.8)$ & $1: 00$ & & $1: 00$ & \\
\hline \multirow{4}{*}{ Referral system } & CVs & $56(74.7)$ & $19(25.3)$ & $1: 00$ & & & \\
\hline & Self-referred & $213(84.2)$ & $40(15.8)$ & $0.553(0.298,1.0298)$ & 0.062 & $0.426(0.210,0.865) *$ & 0.018 \\
\hline & neighbors & $10(76.8)$ & $3(23.1)$ & $0.884(0.220,3.554)$ & 0.862 & $0.532(0.117,2.423)$ & 0.414 \\
\hline & EOS & $7(77.8)$ & $2(22.2)$ & $0.842(0.161,4.408)$ & 0.839 & $0.778(0.121,5.019)$ & 0.792 \\
\hline Distance from & $<30$ minute & $117(82.3)$ & $37(17.7)$ & $0.908(0.524,1.574)$ & 0.732 & & \\
\hline ОТР & $>=30$ minute & $114(80.9)$ & $27(19.1)$ & $1: 00$ & & & \\
\hline \multirow{2}{*}{$\begin{array}{l}\text { Types of } \\
\text { malnutrition }\end{array}$} & Marasmus & $253(82.7)$ & $53(17.3)$ & $1: 00$ & & $1: 00$ & \\
\hline & kwashiorkor & $33(75)$ & $11(25)$ & $1.591(0.756,3.348)$ & 0.221 & $2.536(1.066,6.034) *$ & 0.035 \\
\hline \multirow{2}{*}{ RUFT intake } & Adequate & $275(81.4)$ & $63(18.6)$ & $2.520(0.319,19.878)$ & 0.380 & & \\
\hline & Inadequate & $11(91.7)$ & $1(8.3)$ & 1:00 & & & \\
\hline \multirow{2}{*}{ Appetite test } & Appetite test done & $284(81.8)$ & $63(18.2)$ & $0.444(0.40,4.969)$ & 0.510 & & \\
\hline & Not done & $2(66.7)$ & $1(33.3)$ & $1: 00$ & & & \\
\hline \multirow{2}{*}{ Breast feeding } & Yes & $109(82.6)$ & $23(17.4)$ & $0.911(0.518,1.601)$ & 0.746 & & \\
\hline & No & $177(81.2)$ & $41(18.8)$ & $1: 00$ & & & \\
\hline \multirow{2}{*}{ Diarrhea } & present & $78(80.4)$ & 19 (19.6) & $1.126(0.620,2.043)$ & & & \\
\hline & Absent & $208(82.2)$ & $45(17.8)$ & $1: 00$ & 0.697 & & \\
\hline
\end{tabular}

COR; Crude Odds Ratio, AOR; Adjusted Odds Ratio, EOS; Enhanced Outreach Strategy.

\section{Discussions}

The objective of this study was to explore the treatment outcomes SAM and associated factors among children aged 6-59 months admitted in OTPs sites Gursum woreda selected health facilities. The treatment outcomes of this study were $287(81.7 \%), 44(12.6 \%), 0(0 \%), 3(0.9 \%), 8$ (2.3\%), 3.9 $(2.6 \%), 3.8 \mathrm{~g} / \mathrm{kg} /$ day and 6.7 weeks of recovery rate, defaulter rate, death rate, non-response rate, medical transfer, unknown, weight gain and length of stay respectively. Significantly associated factors were type of malnutrition and referral system. Except average weight gain and average length of stay all others indicators met performance indicator and within the SPHERE Standard rage which is greater than $75 \%$ [19].

There are variation between the findings of the current work and similar studies conducted in Ethiopia, this may be due to variation in characteristics of study participants such as child feeding habit, geographical variation which might influence level of health service utilization, access to nutrition supplies and getting support from partners, availability of health services, life style of the pastoral and agro-pastoralist community may vary and resulted recovery rate difference.

The recovery rate was $(81.7 \%)$ which is higher than studies conducted in different part of the globe that ranges from $10.2 \%$ to $77.9 \%$ [20-27]. Similarly, our current work is in line with another study that found to be $83.2 \%, 85 \%, 87 \%$ for studies conducted in Afar, Southern and Tigray regions respectively $[7,23,28]$. The findings of this study indicated an improvement of recovery rate and no mortality (case fatality) rate was observed children admitted to the OTP sites.

Both death rate and default rate were found to be acceptable range of international SPHRE Standard reference which are $10 \%$ and $15 \%$ respectively [19]. The study also showed a default rate of $(12.6 \%)$ which is below standard reference point; default rate is lower than studies conducted in Yemen which is $80.2 \%$ and $50 \%$ respectively. $[20,26]$. It is higher than studies conducted in afar region, Tigery region and Kenya $6.3 \%, 2.2 \%$ and $2.9 \%$ respectively [23-24, 28].

Comparing other studies conducted different parts of the world this study reported $0 \%$ death rate compared to studies done in Yemen and Afar regions which reported $4.9 \%$ and $3.3 \%$ respectively $[26,28]$. Current study found that length of stay in OTP site for this study was 6.7 weeks which was alarming and not acceptable than minimum international standard of four weeks; yet it is acceptable according to Ethiopian standard protocol management of severe acute malnutrition which is minimum of 8 weeks $[18,29]$. It is also consistent with institutional based studies which were conducted in Southern Ethiopia that showed a range of 8.7 to 6.7 weeks [22-23, 27].

Length to stay for this study is lower than those conducted in Shebedino district, Southern Ethiopia which shows 9 weeks [30]. Similarly there are other studies that were higher than current study which revealed that greater than 10 weeks $[26,28]$. Prolonged length of stay in Gursum district may be due to the fact it is different in socio-demographic, socioeconomic, socio-cultural factors, geographical variation of health care setting, health and access and utilization of health facilities compared to other settings and might be possible 
explanation for this finding.

Overall weight gain of children admitted in to OTP at Gursum woreda health facilities was $3.8 \mathrm{~g} / \mathrm{kg} / \mathrm{day}$ in the program which is out of acceptable range of international SPHERE Standard [31]. The finding was in line with facility based retrospective cohort study done in Shebedido district, Southern Ethiopia which was $3.85 \mathrm{~g} / \mathrm{kg} /$ day [25]. There are studies higher than current study that reported a range of 4.2 to $11.04 \mathrm{~g} / \mathrm{kg} /$ day $[24,27-28]$. Prolonged length of stay and lesser average weight gain in Gursum district may be due to low community awareness since $72.3 \%$ of children were selfreferred. Sharing of therapeutic feeding at household level might another possible explanation.

Children suffering from kwashiorkor were 2.5 times more likely to recover compared to those children who had marasmus (AOR: 2.53, 95\% CI: 1.07, 6.03). Our findings is in agreement with other studies that were conducted in Ethiopia and Ghana which showed that children admitted with kwashiorkor recovered quickly comparing to their marasmic counterparts $[23,28,32]$ but disagree with a finding from Kamba district of Ethiopia which found no association between type of malnutrition and SAM recovery rate [33]. The possible justification lower recovery rate among marasmic children is that there are concurrent of protein energy deficiency and other morbidities compared to children who suffer from kkwashiorkor who only suffer from protein energy deficiency.

Due to the nature of the study design cause-effect relationship of recovery rate and associated factors of severe acute malnutrition is not shown. Further, it is not clear whether allocated RUFT was given as per protocol or not. Most of household factors are not studied like socioeconomic, socio-demographic and other factors that may play a role in child recovery rate.

\section{Conclusion}

The study shown that recovery rate, death rate, default rates were within the acceptable range of sphere standard but still need further effort to improve the expected outcome. The average weight gain, length of stay, self-transfer and children who had kwashiorkor were significantly associated with recovery rate. In summary, health education for caretakers on seeking medical care earlier and, involving more community health volunteers in early case detection and adherence to the treatment protocols may improve SAM treatment outcomes in the government health facilities.

\section{Recommendations}

Regional health bureau should increase community awareness on OTP services available for management severe acute malnutrition. Health workers working at the health posts also need to be capacitated to ensure the quality of treatment services which help the performance indicators are within the SPHERE standard range. Further, more community health volunteers should be trained and involved in community mobilization and active case finding.

\section{References}

[1] Hobbs B and B. A., Acute malnutrition: an everyday emergency; a 10-point plan for tackling acute malnutrition in under-fives.. Generation nutrition campaign report, 2014.

[2] WHO, Updates on the management of severe acute malnutrition in infants and children. 2013, Geneva: World Health Organization.

[3] HTP., Technical notes on management of severe acute malnutrition. Vol. Module 13. 2011.

[4] K., S., Community-based therapeutic care: treating severe acute malnutrition in sub-Saharan Africa. London: University College London, 2010.

[5] Central Statistical Agency Ethiopia, Ethiopia Demographic and Health Survey, and ICF., Central Stastical Agency (Ethiopia), ICF International. Ethiopian Demographic and Health Survey in Rockville, Maryland, USA: CSA and ICF. 2016: Addis Ababa, Ethiopia.

[6] Organization, W. H. and UNICEF, Community-based management of severe acute malnutrition: a joint statement by the World Health Organization, the World Food Programme, the United Nations System Standing Committee on Nutrition and the United Nations Children's Fund. 2007.

[7] Teferi, E., et al., Treatment outcome of children with severe acute malnutrition admitted to therapeutic feeding centers in Southern Region of Ethiopia. Ethiopian Journal of Health Development, 2010. 24 (3).

[8] UNICEF, Evaluation of community management of acute malnutrition (CMAM). Ethiopia: Federal Ministry of Health, Government of Ethiopia and UNICEF Country Office, 2012.

[9] Akparibo, R., et al., Severe acute malnutrition in children aged under 5 years can be successfully managed in a nonemergency routine community healthcare setting in Ghana. Maternal \& child nutrition, 2017. 13 (4): p. e12417.

[10] Kabalo, M. Y. and M. M. Shanka, Seasonal variations of admission and survival status of children treated for severe acute malnutrition (SAM) at outpatient therapeutic program (OTP) in Wolaita Zone, Southern Ethiopia. International Journal of Collaborative Research on Internal Medicine \& Public Health, 2016.8 (9): p. 512-526.

[11] Yebyo, H. G., et al., Outpatient therapeutic feeding program outcomes and determinants in treatment of severe acute malnutrition in tigray, Northern Ethiopia: a retrospective cohort study. Plos one, 2013. 8 (6): p. e65840.

[12] Fanzo, J., et al., 2018 Global Nutrition Report: Shining a light to spur action on nutrition. 2018.

[13] UNICEF, Management of Severe Acute Malnutrition in children. 2015.

[14] UNICEF, Annual Report-Ethiopia. 2013.

[15] Heikens GT, et al., Case management of HIV-infected severely malnourished children: challenges in the area of highest prevalence. lancet, 2008. 7. 
[16] Commission., F. D. R. o. E. P. C., Summary and Statistical report of the 2007 population and housing census. Addis Ababa, Ethiopia.. 2008 Dec.

[17] Kabalo MY and S. CN., Treatment outcomes of severe acute malnutrition in children treated within Outpatient Therapeutic Program (OTP) at Wolaita Zone, Southern Ethiopia: retrospective cross-sectional study. Journal of Health, Population and Nutrition, Dec 201736 (1): p. 7.

[18] E., F., Protocol for the management of severe acute malnutrition Federal Ministry of health. 2007, Addis Ababa.

[19] Golden M and G. Y., Protocol for the management of severe acute malnutrition. MOH. 2007, Ethiopia.

[20] Al Amad, M., et al., Evaluation of outpatient therapeutic programme (OTP) for treatment of severe acute malnutrition in Yemen: a focus on treatment default and its risk factors. Journal of evaluation in clinical practice, 2017. 23 (6): p. 1361-1366.

[21] Asres, D. T., R. P. Prasad, and T. A. Ayele, Recovery time and associated factors of severe acute malnutrition among children in Bahir Dar city, Northwest Ethiopia: an institution based retrospective cohort study. BMC Nutrition, 2018. 4 (1): p. 17.

[22] Fikrie, A., A. Alemayehu, and S. Gebremedhin, Treatment outcomes and factors affecting time-to-recovery from severe acute malnutrition in 6-59 months old children admitted to a stabilization center in Southern Ethiopia: A retrospective cohort study. Italian journal of pediatrics, 2019. 45 (1): p. 46.

[23] Kabalo, M. Y. and C. N. Seifu, Treatment outcomes of severe acute malnutrition in children treated within Outpatient Therapeutic Program (OTP) at Wolaita Zone, Southern Ethiopia: retrospective cross-sectional study. Journal of Health, Population and Nutrition, 2017. 36 (1): p. 7.

[24] Mbaya, D., et al., Outcomes of severely malnourished children aged 6-59 months on outpatient management program in Kitui County hospital, Kenya. Open Journal of Pediatrics, 2015. 5 (04): p. 326.

[25] Mengesha, M. M., et al., Treatment outcome and factors affecting time to recovery in children with severe acute malnutrition treated at outpatient therapeutic care program. Global health action, 2016.9 (1): p. 30704.

[26] Muftah, S., Outpatient management of severe acute malnutrition among children under five years old, in Yemen: a retrospective cohort study. International Journal of Contemporary Pediatrics, 2016. 3 (2): p. 445.

[27] Shanka, N., S. Lemma, and D. Abyu, Recovery rate and determinants in treatment of children with severe acute malnutrition using outpatient therapeutic feeding program in Kamba District, South West Ethiopia. J Nutr Disord Ther, 2015. 5 (2): p. 155.

[28] Liben, M. L., A. G. Wuneh, and R. Shamie, Factors associated with child survival in children admitted to outpatient therapeutic program at public health institutions in Afar Regional State, Ethiopia: a prospective cohort study. Journal of Health, Population and Nutrition, 2019. 38 (1): p. 35 .

[29] Yebyo HG, et al., Outpatient Therapeutic Feeding Program Outcomes and Determinants in Treatment of Severe Acute Malnutrition in Tigray, Northern Ethiopia A Retrospective Cohort Study. PloS one 2013.

[30] Teshome, G., T. Bosha, and S. Gebremedhin, Time-torecovery from severe acute malnutrition in children 659 months of age enrolled in the outpatient treatment program in Shebedino, Southern Ethiopia: a prospective cohort study. BMC Pediatrics, 2019. 19 (1): p. 33.

[31] H., C., Minimum standards in food security and nutrition. In: sphere hand book. 2011.

[32] Saaka, M., et al., Treatment outcome of severe acute malnutrition cases at the tamale teaching hospital. Journal of nutrition and metabolism, 2015. 2015.

[33] Shanka NA, Lemma S, and A. DM., Recovery rate and determinants in treatment of children with severe acute malnutrition using outpatient therapeutic feeding program in Kamba District, South West Ethiopia.. Journal of Nutritional Disorders \& Therapy., 2015. 